Conclusion. The initial audit identified that most patients had IPE, ECG and bloods but this was documented appropriately in less than $42 \%$ had this appropriately documented.

Interventions to improve this rate were developed, focussing on increasing completion of IPE, ECG and bloods as well as improving documentation. The completion of PHIT document is now monitored regularly. The re-audit to identify the magnitude of improvements from these interventions is currently underway.

How can automated linguistic analysis help to discern functional cognitive disorder from healthy controls and mild cognitive impairment?

Lizzie Beavis $^{1 \star}$, Ronan O’Malley ${ }^{2}$, Bahman Mirheidari ${ }^{3}$, Heidi Christensen ${ }^{3}$ and Daniel Blackburn ${ }^{2}$

${ }^{1}$ The University of Sheffield; ${ }^{2}$ Department of Neuroscience, University of Sheffield, Sheffield Teaching Hospitals NHS Foundation Trust, Department of Neurology and ${ }^{3}$ Dept of Computer Science, University of Sheffield; Heidi Christensen, Dept of Computer Science, University of Sheffield

${ }^{\star}$ Corresponding author.

doi: 10.1192/bjo.2021.78

Aims. The disease burden of cognitive impairment is significant and increasing. The aetiology of cognitive impairment can be structural, such as in mild cognitive impairment (MCI) due to early Alzheimer's disease (AD), or in functional cognitive disorder (FCD), where there is no structural pathology. Many people with FCD receive a delayed diagnosis following invasive or costly investigations. Accurate, timely diagnosis improves outcomes across all patients with cognitive impairment. Research suggests that analysis of linguistic features of speech may provide a noninvasive diagnostic tool. This study aimed to investigate the linguistic differences in conversations between people with early signs of cognitive impairment with and without structural pathology, with a view to developing a screening tool using linguistic analysis of conversations.

Method. In this explorative, cross-sectional study, we recruited 25 people with MCI considered likely due to $\mathrm{AD}$, (diagnosed according to Petersen's criteria and referred to as PwMCI), 25 healthy controls (HCs) and 15 people with FCD (PwFCD). Participants' responses to a standard questionnaire asked by an interactional virtual agent (Digital Doctor) were quantified using previously identified parameters. This paper presents statistical analyses of the responses and a discussion of the results.

Result. PwMCI produced fewer words than PwFCD and HCs. The ratio of pauses to speech was generally lower for PwMCI and PwFCD than for HCs. PwMCI showed a greater pause to speech ratio for recent questions (such as 'what did you do at the weekend?') compared with the HCs. Those with FCD showed the greatest pause to speech ratio in remote memory questions (such as 'what was your first job?'). The average age of acquisition of answers for verbal fluency questions was lower in the MCI group than HCs.

Conclusion. The results and qualitative observations support the relative preservation of remote memory compared to recent memory in MCI due to AD and decreased spontaneous elaboration in MCI compared with healthy controls and patients with FCD. Word count, age of acquisition and pause to speech ratio could form part of a diagnostic toolkit in identifying those with structural and functional causes of cognitive impairment. Further investigation is required using a large sample.
N-Methyl-D-Aspartate Receptor binding in First-Episode Psychosis: A PET brain imaging study

Katherine Beck ${ }^{1 \star}$, Atheeshaan Arumuham ${ }^{1}$, Barbara Santangelo ${ }^{1}$, Mattia Veronese ${ }^{2}$, Robert McCutcheon ${ }^{1}$, Stephen Kaar ${ }^{1}$, Colm McGinnity ${ }^{3}$, Toby Pillinger ${ }^{1}$, Faith Borgan ${ }^{1}$, Alexander Hammers ${ }^{2}$ and Oliver Howes ${ }^{1}$

${ }^{1}$ IoPPN King's College London; ${ }^{2}$ IoPPN King's College and ${ }^{3}$ Kings College London

${ }^{\star}$ Corresponding author.

doi: 10.1192/bjo.2021.79

Aims. Evidence from genetics, post mortem and animal studies suggest that N-Methyl-D-Aspartate Receptor (NMDAR) hypofunction has an important role in the pathophysiology of psychosis. However, it is not known if NMDAR activity is altered in the early stages of psychosis or if this links to symptom severity. Our aim was to investigate NMDAR availability in first-episode psychosis (FEP) and determine if it links to symptom severity. The NMDAR hypofunction hypothesis of schizophrenia was initially proposed in the 1990s on the basis of observations that ketamine and phencyclidine (PCP) induced the full range of schizophrenia-like symptoms (positive, negative and cognitive) when given to healthy participants and also that they worsen symptoms in patients with schizophrenia. Method. We recruited 40 volunteers, including 21 patients with schizophrenia from early intervention services in London (12 antipsychotic-free and 9 receiving antipsychotic medication) and 19 matched healthy controls. The uptake of an NMDAR selective ligand, [18F]GE179, was measured using positron emission tomography (PET) and indexed using the distribution volume ratio (DVR) and volume of distribution (VT, in millilitres per cubic centimetre) of $[18 \mathrm{~F}] \mathrm{GE} 179$ in the hippocampus and additional exploratory regions (anterior cingulate cortex (ACC), thalamus, striatum and temporal lobe). Symptom severity was measured using the Positive and Negative Syndrome Scale (PANSS).

Result. A total of 37 individuals were included in the analyses (mean [SD] age of controls, 26.7 [4.5] years; mean [SD] age of patients, 25.3 [4.9] years). There was a significant reduction in hippocampal DVR in the patients with schizophrenia relative to healthy controls $(p=0.02$, Cohen's $d=0.81)$. Although the VT of $[18 \mathrm{~F}]$ GE179 was lower in absolute terms in patients, there was no significant effect of group on VT in the hippocampus ( $p=0.15$, Cohen's $\mathrm{d}=0.49$ ) or the exploratory brain regions. There was a negative association between hippocampal DVR and total PANSS symptoms (rho $=-0.47, \mathrm{p}=0.04$ ), depressive symptoms ( $\mathrm{rho}=-0.67$, $\mathrm{p}=0.002)$, and general PANSS symptoms (rho $=-0.74, \mathrm{p}=0.001)$. Conclusion. These results indicate lower hippocampal NMDAR levels in schizophrenia relative to controls with a large effect size, and that lower NMDAR levels are associated with greater levels of symptom severity. These findings are consistent with the role of NMDAR hypofunction in the pathophysiology of schizophrenia; however, further work is required to test specificity and causal relationships.

\section{Psychosomatic aspects of psoriasis and atopic dermatitis}

Olga Belugina

Belarusian State Medical University

doi: 10.1192/bjo.2021.80

Aims. The aim of this study is to assess the level of alexithymia, coping strategies and stress contribution to illness in patients with 